\title{
Le développement de la TPE camerounaise : évidences ou paradoxes?
}

Jean-François Ngok Evina

\section{OpenEdition}

1 Journals

Édition électronique

URL : http://journals.openedition.org/communicationorganisation/380

DOI : 10.4000/communicationorganisation.380

ISSN : $1775-3546$

Éditeur

Presses universitaires de Bordeaux

Édition imprimée

Date de publication : 1 décembre 2007

Pagination : 150-166

ISSN : 1168-5549

\section{Référence électronique}

Jean-François Ngok Evina, "Le développement de la TPE camerounaise : évidences ou paradoxes ? », Communication et organisation [En ligne], 32 | 2007, mis en ligne le 01 décembre 2010, consulté le 30 avril 2019. URL : http://journals.openedition.org/communicationorganisation/380 ; DOI : 10.4000/ communicationorganisation.380 
Analyses

\section{Résumé}

Suite au développement des technologies de l'Information et de la Communication, la croissance de l'entreprise doit tenir compte des variables non seulement internes, mais de plus en plus externes. L'étude porte sur 140 TPE camerounaises appartenant à divers branches d'activités économiques. Les résultats de cette recherche indiquent que le niveau d'études du dirigeant ainsi que ses aspirations n'ont aucun impact sur le développement de l'entreprise.

\section{Mots-clés}

Très petite entreprise, profil du dirigeant, typologies d'entrepreneurs, Cameroun, croissance.

\section{Abstract \\ Following the development of communication and information technologies, the growth of the company must hold account of the variables not only internal, but increasingly external. The study relates to 140 Cameronian TPE belonging to various branches of economic activities. The results of this research indicate that the level of studies of the leader as its aspirations do not have any impact on the development of the company.}

\section{Keys-Words}

Very Small enterprise, manager profil, typology of entrepreneurs, Cameroon, growth.

Jean François Ngok Evina est enseignant-chercheur à l'IRGO de l'Université Montesquieu Bordeaux IV (France), Chef de Division des Techniques de Gestion Université de Douala Cameroun 


\section{Le développement de la TPE camerounaise : évidences ou paradoxes? \\ Jean-François Ngok Evina,} ngokevina@yahoo.fr

Le Cameroun, pays d'Afrique au sud du Sahara comprend dix régions ou provinces. Notre recherche s'intéresse particulièrement aux grandes métropoles économiques que sont: Douala, Yaoundé, Bafoussam et Garoua. Le choix de ces localités se justifie par l'intensité des transactions économiques effectuées. L'économie camerounaise repose majoritairement sur les PME en général et sur les TPE (structure employant moins de dix salariés) en particulier. La création de ces petites structures permet de réduire le chômage des jeunes et contribue de manière significative au développement locale notamment par le versement de l'impôt libéral qui permet de financer les projets municipaux en l'occurrence, l'électrification en zones urbaine et rurale, la construction des écoles et des hôpitaux pour ne citer que ces réalisations là. Le passage de la TPE à la petite entreprise voire à la moyenne entreprise semble plus rarement étudié. Le vide s'exprime particulièrement dans l'optique d'une compréhension des logiques d'actions des entrepreneurs. En effet, l'angle d'approche des recherches reste descriptif des profils d'entrepreneurs, ce qui permet l'émergence de certaines tendances, mais procure peu d'explications ou de compréhension du phénomène. Quelles sont les variables qui influencent le développement de la TPE camerounaise ? Telle semble être la principale question à laquelle le présent article tente de répondre.

L'objectif de cette recherche est d'expliquer que le développement d'une TPE est lié ou non au profil de son dirigeant. L'article s'articule en trois sections : la première porte sur la revue de la littérature, la seconde concerne la démarche méthodologique adoptée tandis que la troisième présente les principaux résultats de la recherche.

\section{Revue de la littérature}

Suite aux mauvaises performances réalisées par l'économie camerounaise depuis une dizaine d'années, passant ainsi d'un Pays en Voie de Développement (PVD) hier à un Pays Pauvre et Très Endetté (PPTE) aujourd'hui. Ceci étant, sur le plan mondial, nous assistons à la montée en puissance des petites structures très flexibles en l'occurrence les TPE (Marchesnay, 2003). L'attention portée à la petite entreprise en générale et à la TPE en particulier, perçue comme 
Analyses

une forme organisationnelle spécifique, fournit à la recherche un certain nombre d'interrogations nouvelles ou résurgentes (Divay, 1999). Le qualificatif «intermédiaire », couramment appliqué à ces entreprises, induit une réflexion, non seulement sur la situation spécifique de la firme, mais également sur son mode de développement, en l'orientant vers des perspectives longitudinales. Le propriétaire dirigeant de la PME, et plus particulièrement celui de la TPE, exerce une très forte influence sur le système de gestion de son entreprise, soit tout à la fois sur sa structure et son fonctionnement. C'est la raison pour laquelle dans le champ de l'entrepreneurship, plusieurs questions font l'objet de recherches répétées et de nombreuses publications. Plusieurs recherches identifient certaines relations entre dirigeant et entreprise. Par exemple, il semble possible de trouver des réflexions sur les liens entre comportement et stades d'évolution (Lorrain et Dussault, 1994), ou bien sur la relation visionperformance (Nkongolo-Bakenda et alii, 1994), voire sur le lien entre perception manageriale et comportement stratégique (Paradas, 1998). Compte tenu de la multiplicité d'objectifs (Marchesnay, 1997, 1998) et des orientations stratégiques des dirigeants, leurs comportements ne sont pas « naturellement» orientés par une rationalité formelle (c'està-dire managériale). On suppose globalement que, les dirigeants de TPE adoptent des attitudes éloignées, voire contraires à la logique du marché et à la rationalité économique. Les recherches dans le domaine de l'entrepreneuriat et de la PME considèrent l'entrepreneur comme un personnage essentiel au cœur de l'activité entrepreneuriale, et ce, même si le profil des dirigeants d'entreprises est reconnu depuis un certain temps par de nombreux auteurs comme un facteur important quant aux choix stratégiques effectués.

Il convient ainsi d'examiner successivement le développement de l'entreprise, la typologie d'entrepreneur d'une part et les réalités de l'entrepreneuriat en Afrique d'autre part.

\section{Typologie d'entrepreneur et développement de son entreprise}

Chercheurs et consultants établissent des portraits robots de " l'entreprise type » tout au long de son développement. Ils décrivent ainsi trois à cinq stades de développement, depuis celui de la très petite entreprise aux capitaux très limités, à la structure formalisée et décentralisée qu'est la grande entreprise. Or, à travers la vie de 110 petites et moyennes entreprises en croissance, Churchill et Lewis (1983) montrent que le schéma des modèles de métamorphose doit être compris comme une tendance générale d'évolution plutôt que comme une vérité universelle. Plusieurs stades de développement sont 
ainsi identifiés par ces auteurs : le premier stade correspond à la période durant laquelle l'entreprise est généralement gérée quasi exclusivement par son dirigeant, véritable homme à tout faire. Au deuxième stade, la communication devient plus impersonnelle et plus formelle. D'homme à tout faire, le dirigeant devient superviseur. Il ne délègue pour autant que la gestion courante, une grande centralisation étant de rigueur. La croissance de la firme peut alors redémarrer dans de bonnes conditions jusqu'à ce que la structure en place montre à son tour des signes évidents de saturation. Au troisième stade, de nouveaux niveaux hiérarchiques sont créés. La délégation est réelle et s'exerce soit par unité géographique, soit par division. Les différents stades de développement ainsi énoncés sont critiquables et discutables car le passage d'un stade à l'autre est floue et n'est pas linéaire. C'est la raison pour laquelle, Scheib-Bienfait (2003) distingue trois temps dans l'évolution d'une petite entreprise artisanale. Elle opère un découpage artificiel et simplificateur, ce qui confère à cette représentation un caractère linéaire et descriptif. Toutefois, ces trois phases permettent de mettre en correspondance l'évolution des projets entrepreneuriaux des créateurs et les phases effectives de développement de l'entreprise.

Laufer (1975) quant à elle, à partir d'un échantillon de 50 cas de créations d'entreprises qui s'échelonnent entre les années 1950 et 1970, a pour ambition d'identifier les facteurs personnels et socio professionnels qui interviennent dans la décision de créer une entreprise et qui poussent certains à se lancer dans l'aventure. L'étude fait apparaitre un entrepreneur au visage et aux motivations multiples dont la variété s'interprète en fonction de l'expérience humaine et professionnelle de chacun : rapports avec les parents, origine sociale, études et carrières avant la création, motivations liées à la situation professionnelle. A partir des combinaisons de ces éléments, elle établie quatre typologies d'entrepreneurs: le manageur ou l'innovateur, l'entrepreneur propriétaire, l'entrepreneur refusant la croissance et l'entrepreneur artisan.

Miles et Snow (1978) classent les entrepreneurs en fonction de leur stratégie face à l'innovation. Ils distinguent ainsi quatre typologies d'entrepreneurs : les innovateurs pionniers, les innovateurs par action, les adaptateurs suiveurs et les prospecteurs. Miner (1997) offre un excellent exemple en définissant quatre types d'entrepreneurs à partir de la gestion stratégique de leur organisation. Il étudie des variables liées à la gestion des relations internes (relations intra organisationnelles - style de leadership - contrôle - statut de l'autorité), et externes (stratégie relationnelle - ouverture du capital - 
Analyses

partenariat) et également à l'adoption ou non d'une réelle réflexion stratégique. Il distingue quatre types d'entrepreneurs: personnal achiever, manager pur, expert idea generators et super vendeur (Daval et al. 2002).

Norman Smith (1967), partant des travaux de Collins et Moore, élaborent une typologie des entrepreneurs et de leurs entreprises ; il s'attache plus particulièrement à l'analyse des facteurs de survie et de succès qui se trouvent en relation avec différents types d'entrepreneurs et d'entreprises. Il arrive à la distinction de deux types idéaux d'entrepreneurs en prenant pour base, le niveau d'éducation et la formation des entrepreneurs: l'entrepreneur artisan et l'entrepreneur opportuniste. Cette étude reste des plus classiques et, qui plus est vérifiée (Filion, 2000).

Marchesnay (1993) propose une classification fondée sur les aspirations des dirigeants. Celles - ci reposent sur : la recherche de la pérennité de l'affaire, l'indépendance du capital, ou de l'autonomie de décision, la croissance, qu'elle soit proactive ou réactive. Il présente ainsi deux types extrêmes d'entrepreneurs: les PIC (Pérennité Indépendance Croissance) et les CAP (Croissance Autonomie Pérennité). Les PIC sont mus par une logique d'action essentiellement patrimoniale. Les CAP sont mus par une logique de valorisation du capital, plutôt que de l'accumulation. Ils font volontiers travailler l'argent des autres, mais privilégient l'autonomie de la décision. Ils ne souhaitent pas se pérenniser si c'est au détriment de la rentabilité. Ils évoluent plutôt dans des activités jeunes et turbulentes (Paradas, 1998).

Hernandez (2001b) retient comme principaux axes d'analyse la volonté de croissance et celle d'autonomie. Ces deux notions semblent essentielles pour classer les créateurs, voire pour en établir une typologie. Elles apparaissent comme les plus pertinentes, celles qui permettent le mieux d'établir des catégories signifiantes dans l'univers foisonnant des créateurs. La volonté de croissance oppose ceux pour lesquels l'entrepreneuriat est avant tout un moyen de créer son propre emploi, et ceux qui veulent véritablement construire une organisation. Le désir d'autonomie oppose ceux qui privilégient leur indépendance, ceux pour lesquels être son propre patron est surtout un moyen de ne plus avoir de patron, et ceux pour lesquels cette motivation étant plus secondaire sont plus ouverts à l'idée de partager leur capital, même de devenir minoritaires dans leur propre affaire. Le croisement de ces deux axes fait ressortir quatre profils - types d'entrepreneurs : le manageur, l'exclu, l'entrepreneur et l'artisan. 


\section{Les réalités de l'entrepreneuriat en Afrique}

Contamin (1990) identifie trois typologies d'entreprises en Côte d'Ivoire : les petits métiers, l'artisanat et les PME. Cette typologie se distingue par les différentes étapes du processus d'accumulation. Les artisans n'ont pas exercé de petits métiers avant de démarrer leur activité, ils ne deviennent pas dirigeants de PME. Ces trois niveaux de la petite production marchande restent cloisonnés les uns par rapport aux autres. Aucun phénomène de transition ne permet le passage d'un niveau à l'autre.

Ngok Evina (2002), à partir d'une étude portant sur les entrepreneurs des TPE du Grand-Nord Cameroun identifie six catégories d'entrepreneurs : l'entrepreneur « autodidacte » ; l'entrepreneur « scolarisé »; l'entrepreneur « noble »; l'entrepreneur « chercheur »; l'entrepreneur « imitateur » et l'entrepreneur « innovateur ». Les critères de distinction retenus étant : le niveau d'éducation, l'aversion pour le risque et la croissance.

De l'analyse des différents travaux ci-dessus, les hypothèses suivantes sont retenues :

Hypothèse 1: le niveau d'éducation du dirigeant influence significativement le développement de son entreprise.

\section{Hypothèse 2: les aspirations du dirigeant déterminent le développement de l'entreprise.}

La première partie de cet article nous a permis d'examiner les différentes phases de développement de l'entreprise, de faire le point sur la typologie de l'entreprise et de brosser à grands traits les réalités de l'entrepreneuriat en Afrique. Il convient en outre, de passer à la phase empirique issue des observations de terrain dans un échantillon de TPE du grand nord Cameroun.

\section{Démarche adoptée}

\section{Collecte et traitement des données}

La méthode de collecte des données est l'enquête par questionnaire avec administration directe. Cette méthode permet de limiter les biais lors du traitement des données. La paternité de ce questionnaire revient à Daval, Deschamps et Geindre (2002) qui identifient plusieurs typologies d'entrepreneurs à partir de leurs logiques d'action (ceux qui optent ou non pour la croissance de leur entreprise). Le questionnaire comprend majoritairement des questions fermées et des échelles d'attitude de type Likert à cinq points allant de 1) pas du tout 
Analyses

d'accord à 5) tout à fait d'accord. La cohérence interne des items est mesurée par le coefficient alpha de Cronbach.

L'échantillon de la présente recherche est composé de 140 entrepreneurs (c'est - à - dire, créateurs, propriétaires, dirigeants) qui gèrent directement ou indirectement une TPE. Cet échantillon est représentatif de la population totale parce que toutes les branches d'activités issues de la direction de la statistique et de la comptabilité nationale sont présentent.

La collecte des données proprement dite s'est faite en deux étapes :

1) Nous avons consulté les fichiers des entreprises relevant des mairies des chefs lieux des provinces du littoral, du grand nord, du centre et de l'ouest Cameroun.

\begin{tabular}{|l|l|l|l|l|l|}
\hline & $\begin{array}{l}\text { Grand } \\
\text { nord }\end{array}$ & Centre & Ouest & Littoral & Total \\
\hline $\begin{array}{l}\text { Questionnaires } \\
\text { déposées }\end{array}$ & $\begin{array}{l}20 \\
(11 \%)\end{array}$ & $38(21 \%)$ & $\begin{array}{l}52 \\
(29 \%)\end{array}$ & $70(39 \%)$ & 180 \\
\hline Non réponse & $\begin{array}{l}14 \\
(35 \%)\end{array}$ & $\begin{array}{l}07 \\
(17,5 \%)\end{array}$ & $\begin{array}{l}12 \\
(30 \%)\end{array}$ & $\begin{array}{l}07 \\
(17,5 \%)\end{array}$ & 40 \\
\hline $\begin{array}{l}\text { Questionnaires } \\
\text { exploitables }\end{array}$ & $06(4 \%)$ & $31(22 \%)$ & $\begin{array}{l}40 \\
(29 \%)\end{array}$ & $63(45 \%)$ & 140 \\
\hline
\end{tabular}

Tableau 1 : Dépouillement statistique du questionnaire

$\mathrm{Au}$ total nous avons contacté 180 dirigeants de TPE et en retour, nous avons obtenu 140 réponses exploitables soit un taux de réponse de $78 \%$.

2) Une pré-enquête a été effectuée auprès des propriétaires dirigeants des TPE de la ville de douala afin d'examiner la perception du questionnaire du point de vue des enquêtés.

Le traitement des données est fait par le logiciel SPSS. Compte tenu non seulement de l'objectif poursuivi mais aussi de la nature des variables (essentiellement qualitatives) et de la taille de l'échantillon, l'analyse typologique et l'analyse de variance sont retenues dans le cadre de cette recherche.

Les méthodes de classification ou de typologie ont pour but de regrouper les individus en un nombre restreint de classes homogènes. Il s'agit donc de décrire les données en procédant à une réduction du nombre d'individus. On distingue ainsi deux types de classification : les méthodes non hiérarchiques qui produisent directement une partition en un nombre fixé de classes et les méthodes hiérarchiques 
qui produisent de partitions en classes de plus en plus vastes.

Dans la première méthode, il est à peu près exclu de trouver la meilleure partition possible et il faut se contenter d'un algorithme qui aboutit à des solutions rapprochées tandis que dans la seconde méthode, le principe consiste à construire une suite de partition en $\mathrm{n}$ classes ; n-1 classes; n-2 classes, ... emboîtées les unes aux autres. C'est la seconde méthode qui est retenu dans cet article. Le principal problème consiste à définir le critère de regroupement de deux clases, ce qui revient à définir une distance entre classe. La méthode d'agrégation utilisée est la méthode de «Ward». Elle permet d'obtenir des groupes tels que l'inertie interclasses soit forte et l'inertie intraclasses faible (Bouroche et Saporta, 1980).

L'analyse typologique a l'avantage de séparer un échantillon en plusieurs groupes considérant non seulement la moyenne mais aussi la variance des variables critères (El Louadi, 1995). Le nombre de groupe à retenir est fonction de l'écart entre les différents clusters. Plus l'écart entre deux variables est grand plus le nombre de groupe augmente. Ainsi, dans le cadre de cette recherche, le nombre de groupe retenu à l'aide de cette méthode est trois, car après cette opération, l'écart entre les différentes variables est marginale.

\subsection{Caractéristiques de l'échantillon}

Plus de $27 \%$ d'entrepreneurs ont un niveau d'étude supérieur. Ce faible taux traduit la prise en compte dans ce milieu d'autres facteurs d'évolution de la société autres que l'éducation. Le développement d'un pays en général et d'une région en particulier est lié à l'instruction de sa population. Aussi, les élites mènent une campagne de sensibilisation sur la nécessité d'envoyer les enfants à l'école afin de défendre leur image de marque dans la sphère publique. Brenner, Fouda et Toulouse (1990) considèrent qu'au Cameroun 50\% des entrepreneurs enquêtés ont un niveau d'étude supérieur. Dzaka et Milandou (1994) dans une étude portant sur les entrepreneurs congolais, considèrent que moins de $50 \%$ d'entrepreneurs ont un niveau d'étude supérieur. La différence entre nos résultats et ceux trouvés par ces auteurs résident au fait que leurs travaux portent essentiellement sur les grandes villes : douala pour les premiers et brazzaville pour les seconds.

Dans notre étude, la majorité des entrepreneurs ont moins de 30 ans (61\%). Toutefois, ces résultats se rapprochent de l'âge moyen des patrons du secteur " garages artisanaux » à Abidjan qui est de 30 ans et de ceux obtenus par Braakhuis dans son enquête auprès des agents 
Analyses

de distribution des activités informelles à Yaoundé où l'âge moyen des chefs d'entreprises est de 34 ans. Cette caractéristique montre que les entrepreneurs se lancent plus vite en affaire. La principale raison à cela est sans doute le développement de la culture d'entreprise qui caractérise les jeunes camerounais à l'heure où le pays cherche à atteindre le point d'achèvement de l'initiative PPTE (pays pauvre très endetté).

$81 \%$ des dirigeants sont des hommes ; ce résultat n'est pas très différent de celui obtenu dans une enquête faite par Brenner, Fouda et Toulouse (1990) sur les entrepreneurs Bamilékés de Douala et leur entreprise dans laquelle les auteurs relèvent l'existence de $90 \%$ d'hommes dans l'échantillon des entrepreneurs. Tsika (1990), dans une étude portant sur les petits entrepreneurs brazzavillois trouve que les hommes représentent $60 \%$ de l'échantillon. L'une des dimensions quelque peu paradoxale de la société traditionnelle en Afrique noire est la féminité. La nette différenciation de l'homme et la femme conduit à une domination de l'homme comme observé dans nombre de pays du Sud. Le pouvoir et l'autonomie des femmes sont une utopie. Peut-être, l'explication vient-elle du rôle de la religion musulmane qui considère que la femme doit tout attendre de l'homme. Kamdem (1996) considère que les rapports hommes - femmes dans l'organisation constituent un autre domaine intéressant d'analyse des abus " culturaliste » dans les pratiques de management en Afrique. L'image de la femme dans la société africaine (aussi bien traditionnelle que moderne) est faite d'une double relation d'attachement et de dépendance dans laquelle la société masculine cherche à maintenir le second sexe. Ce paradoxe explique le comportement ambivalent de la femme africaine dans la société actuelle, comportement que Barbier (1985, cité par Kamdem), traduit dans les termes suivants : «mères pacifiques » et « femmes rebelles».

Les entrepreneurs étudiés n'ont pas une grande expérience professionnelle $44,3 \%$ en possèdent, c'est - à - dire qu'ils ont soit travaillé dans une entreprise auparavant soit effectué des études en gestion des entreprises. Ce taux est caractérisé notamment par les anciens fonctionnaires et quelques diplômés de l'enseignement supérieur

La majorité $(71,4 \%)$ des entrepreneurs adopte des techniques de gestion traditionnelles. Cette disposition est évidente dans la mesure où la majorité n'a pas franchi le seuil de l'enseignement supérieur ou d'une école spécialisée en gestion. Ces techniques sont considérées comme traditionnelles car elles n'ont rien de commun avec les 
techniques modernes (direction par objectif, direction participative par objectif, etc.). En fait l'entrepreneur, dans sa gestion quotidienne, tente de concilier deux conceptions totalement opposées : la primauté de l'économique sur le social (société moderne) et la primauté du social sur l'économique (société traditionnelle). De son aptitude à savoir concilier ces exigences contradictoires dépend la survie de son entreprise (Hernandez, 2001a).

Le capital personnel est détenu à 52,1\% par certains entrepreneurs. Celui - ci provient des économies personnelles, des dons ou de l'héritage. Cette catégorie d'entrepreneurs est moins dépendante de l'extérieur car elle détient le capital et le gère librement selon ses convenances. Quelle que soit la forme de l'entreprise, les ressources propres de l'entrepreneur (ou de l'entreprise lorsque celle-ci est créée) constitue l'essentiel du financement. Sur l'ensemble des cas étudiés par Contamin (1990) en Côte d'Ivoire, il considère que l'épargne préalable constitue le principal mode de constitution du capital initial de l'artisanat et des PME modernes. 33\% d'entrepreneurs ont recours aux tontines. Ainsi, Light (cité par Brenner, Fouda et Toulouse, 1990) rapporte que la pratique des clubs d'épargne du genre des tontines explique les différents taux de représentation des groupes ethniques japonais, chinois, noirs américains et noirs caraïbes dans le petit commerce aux Etats-Unis. Ces études indiquent l'importance de certaines variables comme l'importance du réseau ethnique ainsi que le recours aux tontines dans le processus de création d'entreprises.

Certains entrepreneurs $(57,9 \%)$ ont un projet mature. Cela signifie qu'ils ont conçu l'idée de créer leur entreprise en examinant tous les contours de l'acte entrepreneurial. Ils ressortent les avantages et les inconvénients, le domaine d'activité ainsi que les risques liés au métier.

D'autres entrepreneurs $(42,1 \%)$ ont un esprit de compétition. Avoir un esprit de compétition c'est faire face à des difficultés inhérentes à votre activité. Aussi, plusieurs entrepreneurs adoptent des stratégies différentes pour faire face à la concurrence. Par contre, $72,9 \%$ des entrepreneurs ont confiance en eux. En créant une entreprise, il faut d'abord avoir confiance en soi afin de susciter la confiance des autres. Tandis que $8,6 \%$ des entrepreneurs aiment le risque et estiment aisément selon un adage bien connu du milieu « qui ne risque rien n'a rien ». Selon Ponson (1990), le contrôle de l'incertitude est faible en Afrique en comparaison aux pays latins où la sécurité est vivement recherchée, que ce soit à travers les institutions ou la religion. L'enquête réalisée par Dzaka et Milandou (1994) au Congo révèle 
Analyses

deux attitudes de la part des hommes d'affaires, qui indiquent des degrés différents de sensibilité vis-à-vis du risque (au Congo, les entrepreneurs ont peur de la sorcellerie).

Le besoin de sécurité anime 49,3\% des entrepreneurs car ils recherchent un revenu pour leur survie ainsi que de leur famille. Dans le même ordre d'idée, $12,1 \%$ ont un besoin de reconnaissance ils optent pour leur statut personnel et l'estime de soi ; par contre, $27,9 \%$ ont un besoin d'autonomie et $10,7 \%$ enfin ont un besoin de réalisation de soi.

En effet, plus de la moitié des dirigeants sont mariés. Ce résultat est sensiblement le même que celui obtenu par Nihan et Demol dans leur étude sur les entreprises du secteur non structuré moderne de Yaoundé où $53 \%$ des chefs d'entreprises sont mariés. L'origine sociale joue certainement un rôle important dans la direction d'une entreprise en général et de la TPE en particulier. Plusieurs études africaines montrent un pourcentage élevé des enfants ayant des parents commerçants ou exerçant une activité professionnelle libérale dans le domaine commerciale. Ainsi, Filion (1997) montrent que l'attachement aux rôles traditionnels et l'attachement à l'idéal d'entrepreneur influencent fortement la réaction et le comportement des femmes entrepreneurs. Ceci démontre bien que la manière avec laquelle le dirigeant d'une TPE est éduqué et l'influence de ses origines guident généralement la façon de diriger sa TPE car il n'y a aucun caractère et aucun comportement ex nihilo.

A la création de leur entreprise, 39\% de TPE ont moins de trois employés. C'est généralement le cas des entrepreneurs autodidactes ou ceux qui travaillent avec l'aide de la famille. Par contre, $30 \%$ de TPE ont un effectif compris entre sept et dix employés. Ceci étant, les micro-entreprises qui en majorité ne présentent pas, comme le rappelle Maryynczak (cité par Dzaka et Milandou, 1994), les caractéristiques «d'entités entrepreneuriales», c'est-à-dire des entités innovantes, dont la prise de décision est indépendante, la gestion de type rationnel ayant un objectif explicite de profit et de croissance, ne deviennent des $\mathrm{PE}$ voire des PME qu'à titre exceptionnel, car l'objectif privilégié dans ces cas semble être l'auto-création d'emploi résultant de la montée spectaculaire du chômage.

49\% des TPE étudiées ont pour activité principale le commerce. Cette activité ne nécessite pas suffisamment des moyens importants en terme de capital. Généralement ont retrouve les vendeurs à la sauvette. Le secteur le moins représenté ici est l'agriculture. $11 \%$ de TPE optent pour l'agriculture, les membres se sont constitués en GIC 
(Groupement d'Initiative Commune), en coopérative au service des besoins de la communauté. $77 \%$ d'entrepreneurs établissent des objectifs car cet acte permet d'éviter une navigation à vue. Dans la même lignée, $48 \%$ d'entrepreneurs élaborent des plans, la planification est essentiellement orientée vers la planification opérationnelle. Aucune véritable planification stratégique n'est identifiée dans ces TPE. 23\% d'entrepreneurs pratiquent le contrôle de leurs activités. Le contrôle ici est plus orienté au contrôle des activités car sur le plan financier, chaque entrepreneur est le principal responsable.

\subsubsection{Le développement des TPE camerounaises}

Les questions portant sur le développement de l'entreprise concernent uniquement l'effectif du personnel. Celui-ci varie ainsi de 1) pour un effectif inférieur à trois employés ; 2) pour un effectif compris entre 4 et 6 employés et 3 ) pour celui compris entre 7 et 10 employés. Quant à la projection de l'effectif dans cinq ans, les numéros représentent respectivement : 1) pour moins de 10 salariés ; 2) effectif compris entre 10 et 49 ; 3) effectif compris entre 50 et 499 et 4 ) pour un effectif allant de 500 et plus. Les principaux résultats sont résumés dans le tableau ci-dessous :

\begin{tabular}{|l|l|l|l|l|l|l|}
\hline $\begin{array}{l}\text { Nombre } \\
\text { d'employés }\end{array}$ & Moyenne & Médiane & Mode & Min & Max & $\begin{array}{l}\text { Ecart } \\
\text { type }\end{array}$ \\
\hline A la création & 1,879 & 2 & 2 & 1 & 3 & 0,772 \\
\hline Actuel & 2,329 & 2 & 3 & 1 & 3 & 0,683 \\
\hline $\begin{array}{l}\text { Projection } \\
\text { dans 5 ans }\end{array}$ & 2,493 & 3 & 3 & 1 & 4 & 0,978 \\
\hline
\end{tabular}

Tableau 1 : Evolution du nombre d'employés depuis la création

L'effectif moyen à la création est sensiblement compris entre 4 et 6 employés. C'est d'ailleurs la tranche la plus observée car le mode est de 2. Actuellement, il y a eu un accroissement général de la taille des entreprises étudiées car l'effectif moyen au moment de l'enquête est de 2, mais la classe modale est de 3 ce qui représente un effectif compris entre 7 et 10 employés. Les dirigeants des entreprises étudiées envisagent l'accroissement de l'effectif de leur personnel dans cinq ans. Ils estiment que celui-ci atteindra la tranche comprise entre 50 et 499 employés. Ce qui fera passer ces firmes de la TPE à la moyenne entreprise en passant évidemment par la petite entreprise. 
Analyses

\section{Typologie d'entrepreneurs des TPE camerounaises}

Afin de ressortir les typologies d'entrepreneurs, nous avons entrepris de diviser l'échantillon en fonction des caractéristiques propres à l'entrepreneur et à son entreprise. Comme il n'était pas possible d'utiliser simultanément les médianes ou les moyennes de ces caractéristiques comme critères de division, nous avons eu recours à l'analyse typologique.

Les résultats de cette analyse se présentent dans le tableau ci dessous.

\begin{tabular}{|c|c|}
\hline Typologies & Caractéristiques \\
\hline $\begin{array}{l}\text { Entrepreneur } \\
\text { Bâtisseur: } \\
\mathrm{N}=\end{array}$ & $\begin{array}{l}\text { Besoin d'autonomie ; activité principale service ; } \\
\text { financement bancaire ; TPE créés avant } 1995 \text {; niveau } \\
\text { d'étude primaire ou secondaire; recherche la croissance de } \\
\text { leur TPE ; maturité du projet ; effectif actuel des employés } \\
\text { compris entre } 4 \text { et } 6 \text {; nombre d'employés à la création } \\
\text { moins de trois ; entrepreneur de sexe féminin ; aime le } \\
\text { risque ; entrepreneur, propriétaire - dirigeant ; entrepreneur } \\
\text { âgé de moins de } 30 \text { ans. }\end{array}$ \\
\hline $\begin{array}{l}\text { Entrepreneur } \\
\text { Prestige : } \\
\mathrm{N}=\end{array}$ & $\begin{array}{l}\text { Besoin de reconnaissance et de sécurité ; activité principale } \\
\text { artisanat et commerce ; esprit de compétitivité ; confiance } \\
\text { en soi ; effectif actuel des employés compris entre } 7 \text { et } 10 \text {; } \\
\text { nombre d'employés à la création compris entre } 4 \text { et } 6 \text {; } \\
\text { financement par les tontines; l'entrepreneur ne recherche } \\
\text { pas la croissance. }\end{array}$ \\
\hline $\begin{array}{l}\text { Entrepreneur } \\
\text { Dynamique : } \\
\mathrm{N}=\end{array}$ & $\begin{array}{l}\text { Besoin de réalisation ; activité principale agriculture; } \\
\text { financement personnel (fonds propres); TPE crées après } \\
1995 \text {; l'entrepreneur ne recherche pas la croissance ; } \\
\text { effectif actuel des employés moins de trois ; nombre } \\
\text { d'employés à la création compris entre } 7 \text { et } 10 \text {; } \\
\text { l'entrepreneur est un dirigeant-salarié; pratique des } \\
\text { méthodes de gestion traditionnelles. }\end{array}$ \\
\hline
\end{tabular}

Tableau 2 : Profil des entrepreneurs du Grand Nord Cameroun

\section{Tests d'hypothèses}

En ce qui concerne les deux hypothèses, la variable à expliquer est le développement de l'entreprise et la variable explicative est le niveau d'études du dirigeant en ce qui concerne la première hypothèse et ses aspirations pour la seconde. Les résultats du test de cette hypothèse sont présentés dans le tableau ci-après : 
Le développement de la TPE camerounaise...

\begin{tabular}{|l|c|c|c|c|c|c|}
\hline Hypothèses & $\begin{array}{c}\text { Source de } \\
\text { variation }\end{array}$ & $\begin{array}{c}\text { Degré de } \\
\text { liberté }\end{array}$ & $\begin{array}{c}\text { Somme des } \\
\text { carrés }\end{array}$ & $\begin{array}{c}\text { Carrés } \\
\text { moyens }\end{array}$ & $\mathrm{F}$ & $\begin{array}{c}\text { Signifi- } \\
\text { cation } \\
\text { du F }\end{array}$ \\
\hline $\begin{array}{l}\text { Niveau } \\
\text { d'études du } \\
\text { dirigeant }\end{array}$ & Intergroupes & 1 & 0,1079 & 0,1079 & 0,1121 & 0,7383 \\
\cline { 2 - 7 } & Intragroupes & 138 & 132,884 & 0,9629 & & \\
\hline $\begin{array}{l}\text { Aspirations } \\
\text { du dirigeant }\end{array}$ & Intergroupes & 2 & 0,2522 & 0,1261 & 0,1301 & 0,8781 \\
\cline { 2 - 7 } & Intragroupes & 137 & 132,7407 & 0,9689 & & \\
\hline
\end{tabular}

Tableau 3 : Test des hypothèses

Pour valider ou invalider ces hypothèses, il faut que les principaux résultats soient significatifs, c'est-à-dire que le seuil de signification soit sensiblement égal à zéro. Le test n'est pas significatif pour les deux hypothèses. Ces dernières sont ainsi non validées. L'hypothèse 1 stipule que: le niveau d'études du dirigeant influence significativement le développement de son entreprise. Pour tester cette hypothèse, nous avons eu recours à l'analyse de la variance. L'hypothèse 2 stipule que : les aspirations du dirigeant déterminent le développement de son entreprise.

\section{Conclusion}

L'objectif fixé dans cet article est d'essayer de ressortir un lien entre un type d'entrepreneurs et le développement de sa TPE. Trois catégories d'entrepreneurs sont identifiées dans cet article. Il s'agit de l'entrepreneur " Bâtisseur », "Prestige » et " Dynamique ». Deux hypothèses sont à la base de ce travail: hypothèse 1 : le niveau d'études du dirigeant influence significativement le développement de son entreprise et l'hypothèse 2: les aspirations du dirigeant déterminent le développement de l'entreprise. Les différents tests d'hypothèses effectués conduisent à l'invalidation de ces dernières. En ce qui concerne la première hypothèse, Norman Smith (1967) considère l'entrepreneur « opportuniste », plus instruit, mieux intégrer socialement est capable de développer son entreprise. Partant des résultats de notre recherche et des constatations faites sur le terrain, les travaux de Norman Smith sont remis en cause car, le niveau d'éducation n'a aucun impact sur le développement de l'entreprise. Plusieurs hommes d'affaires camerounais sont généralement autodidactes. Les résultats de cette recherche ont cependant certaines limites. D'abord, il faut dire que cette étude est menée après la vague de fermeture d'entreprises privées et la réduction d'effectif à la fonction publique suite à la crise économique qui a secoué fortement notre pays depuis l'année 1987. Les données ont été recueillies au cours d'une période particulièrement difficile. Ensuite, la non-prise en 
Analyses

compte des facteurs environnementaux et des phénomènes tels que les réseaux constituent bien évidemment une limite au travail proposé. L'influence de l'environnement proche de la TPE est souvent déterminante. Bien sûr, le propos ici est de se pencher plus précisément sur les variables de l'organisation et de mettre de côté les variables d'environnement. Pour ne pas éluder totalement cet aspect, il convient de savoir que la TPE est très influencée par la place qu'elle a dans l'environnement concurrentiel, juridique, et économique.

Enfin, il faut remarquer que la nature même des variables étudiées constitue en soi une limite aux résultats de cette recherche. En effet, la vision stratégique du dirigeant d'une TPE est une variable peu tangible. Sa saisie plus complète aurait peut-être nécessité une longue présence du chercheur dans l'entreprise. Par ailleurs, la volonté de croître est mesurée par une approche subjective. Compte tenu des limites qui viennent d'être énumérées, il s'avère utile que la présente étude soit répétée sur une période plus longue tant sur les mêmes entreprises que sur les entreprises d'autres régions. Dans ce cadre, l'approche par les études de cas semble plus appropriée.

\section{Bibliographie}

Billard L., Boissin J-P. et Deschamps B., " Profil du dirigeant et représentation des mécanismes du gouvernement d'entreprises ", Revue des Sciences de Gestion, Direction et Gestion, 2004, n²04, p. 35-47.

Bouroche J-M. et Saporta G., L'analyse des données, Paris, PUF, 1980.

Brenner G.A, Fouda H. et Toulouse J-M., Les tontines et la création d'entreprises au Cameroun, Ed. Aupelf Uref , 1990, p. 97105.

Churchill N.C. et Lewis V.L., "The five stages of small business growth », Harvard Business Review, mai - juin, 1983, p. 30-50.

Contamin B., Développement et financement des PME en Côte d'Ivoire, Ed. Aupelf Uref, 1990, p. 115-127.

Daval, Deschamps H. B. et Geindre S., «Proposition de grille de lecture des typologies d'entrepreneurs $», 1^{\text {er }}$ colloque de l'académie entrepreneuriat, Bordeaux, 2002.

Divay M., " Comportement de croissance des entreprises de taille moyenne. Une étude empirique à partir de 1411 PME basnormandes », Gestion 2000, vol. 16, n6, novembre, 1999, p. 59-80. 
Dzaka-Kikouta T., «La formation de l'esprit d'entreprise chez les Bakongo d'Afrique centrale: une analyse du processus d'émergence des entrepreneurs par la confiance et les réseaux sociaux », Revue Congolaise de Gestion, ${ }^{\circ}$ 8, janvier-juin, 2004, p.37-68.

Dzaka-Kikouta T. et Milandou M., «L'entrepreneuriat congolais à l'épreuve des pouvoirs magiques: une face cachée de la gestion culturelle du risque », Politique africaine, $\mathrm{n}^{\circ}$ 56, décembre, 1994, p.108-118.

El Louadi M., "L'effet de la congruence informationnelle sur la performance financière des petites banques américaines », Revue Internationale PME, vol. 8, $\mathrm{n}^{\circ} 1,1995$, p. 31-56.

Filion L.J, «Six types de propriétaires - dirigeants de PME », Revue Organisation et Territoires, vol. $9, \mathrm{n}^{\circ} 1$, hiver 2000, p. 5-18.

Filion, L.J., « Le champ de l'entrepreneuriat : historique, évolution, tendances », Revue Internationale PME, volume 10, n², 1997, p. 129 $-172$.

Hernandez E.M., "L'entrepreneur informel africain et la démarche marketing », Recherche et Applications en Marketing, vol. X, n³95, 2001a, p. 47-61.

Hernandez E.M., L'entrepreneuriat: Approche théorique, Paris, L'Harmattan, 2001b

Julien P-A. et Marchesnay M., L'entrepreneuriat, Paris, Economica, 1996.

Kamdem, E., « Nouveau regard sur les pratiques de management au Cameroun », (in) Organisations économiques et cultures africaines. De l'homo oeconomicus à l'homo situs, Paris, L'Harmattan, 1996, p. 249 - 271.

Laufer J., «Comment on devient entrepreneur », Revue Française de Gestion, novembre - décembre, 1975, p. 11-26.

Lorrain J. et Dussault L., «Les entrepreneurs artisans et opportunistes : une comparaison de leurs comportements de gestion », Revue Internationale PME, vol. $7 \mathrm{n}^{\circ}$ 1, 1994, p. 157- 169.

Marchesnay M., «La moyenne entreprise existe-t-elle? », Revue Française de Gestion, n ${ }^{\circ} 116,1997$, p. 85-94.

Marchesnay M., "Confiances et logiques entrepreneuriales », Economies et Sociétés, Série sciences de gestion, 1998, $\mathrm{n}^{\circ}$ 8-9.

Marchesnay M., «La petite entreprise : sortir de l'ignorance », Revue 
Analyses

Marchesnay M., " De l'hypofirme à l'hypogroupe », Cahiers du Lebass, 1993, p. 33 - 50.

Miles R.E et Snow C.C., Organisational strategy structure ans process, Mc Graw Hill, 1998.

Ngok Evina J-F., « Vers une typologie d'entrepreneurs dans les TPE du Grand Nord Cameroun », Actes du Séminaire International du Réseau Africain et Malgache pour l'Excellence en Gestion des Entreprises (RAMEGE), 2002, mai, pp.53-68.

Nkongolo-Bakenda J.M., D'Amboise G. et Garnier B., «L'association entre la manifestation de la vision stratégique des propriétaires dirigeants et la performance de leur entreprise », Revue Internationale PME, vol. 7, n¹, 1994,p. 35-61.

Norman Smith R., «The entrepreneur and his firm the relationship between type of man and type of company », East Lansing Michigan, Michigan State University, 1967.

Paradas A., "L'influence de la personnalité du dirigeant sur le système de gestion de la TPE : utilisation conjointe de deux méthodes adaptées à l'étude de la TPE », Economies et Sociétés, 1998, n², p. 7-30.

Ponson B., Individualisme ou communauté : quelques implications managériales pour l'entreprise en Afrique, Ed. Aupelf Uref, 1990, p. $15-25$.

Scheib-Bienfait $\mathbf{N}$., «L'analyse stratégique à l'épreuve des faits : étude de cas d'une petite entreprise en mutation ", Revue Internationale PME, vol. 16,2003, n 1 , p. 133-163.

Siu W.S., « Entrepreneurial typology: The case of owner - managers in China », International Small Business Journal, 14, 1, 1985, pp. 53 64.

Traoré B., La dimension culturelle de l'acte d'entreprendre en Afrique, Ed. Aupelf Uref, 1990, p. 7-14.

Tsika J., L'esprit d'entreprise: un produit de l'histoire le cas des petits entrepreneurs brazzavillois, Ed. Aupelf Uref, 1990, p. 311-322. 
